\title{
LA UTILIZACIÓN DE ENTORNOS VIRTUALES EN LA FORMACIÓN DE TECNÓLOGOS.
}

\author{
Autor. \\ María L. Rodríguez \\ Profesora de la Universidad de \\ Oriente \\ mariluz@uo.edu.cu
}

\author{
Coautor. \\ León C. Manuel \\ Profesor Titular del ITB \\ manuel_leonc@hotmail.com
}

\author{
Coautor. \\ Emma Z. Delgado \\ Profesora Titular del ITF \\ zulayd@formacion.edu.ec
}

País Origen: Ecuador

Palabras claves: Superación, recursos digitales, profesionales.

Keywords: learning, digital sources, profesional.
Recibido: 11 de Marzo del 2016

Aceptado: 15 de Abril del 2016

\section{SUMMARY}

$\mathrm{T}$ he teaching learning process in higher education requires changes to potentiate the quality and warmth that demands Ecuador from the Organic Law for this educational system. As consequence the objective of this work is to synthesise the different researches about the NTT and its contextualization in order to favour the professional proficiency of teachers. The use of digital sources is remarkable in technological schools because the society demands a professional competence that could satisfy the social needs. From this perspective professors should be prepared to promote the use of the TICs as important goals and didactic resources in the learning process of the students and their future preparation as professionals. The application of interviews, surveys, and other instruments to teachers and students corroborated the difficulties manifested in the use of the NNT in the teaching learning process. Subsequently, to analyze the main theoretical referents permit to design the preparation of teachers in order to promote a higher quality in their performance.

\section{RESUMEN}

I proceso de enseñanza aprendizaje en la educación superior, requiere cambios que potencien la calidad y calidez que se exige en Ecuador desde la Ley Orgánica para este sistema educativo. Por tanto se determina como objetivo: sistematizar los diferentes estudios científicos acerca de las TIC,s y su contextualización, de modo que favorezcan el mejor desempeño profesional de los docentes. La potenciación del uso de los recursos digitales es de vital importancia en los tecnológicos, porque la sociedad exige a los graduados una competencia profesional que responda a las exigencias sociales. Desde esta óptica el docente debe estar preparado para promover el uso de las TIC como fin y recurso didáctico de gran importancia en el aprendizaje de los estudiantes y en su preparación futura como profesionales. La utilización de entrevistas, encuestas y otros instrumentos a docentes y estudiantes, posibilitaron corroborar las dificultades que se manifiestan en la utilización de las TIC,s en el proceso de enseñanza aprendizaje. Por tanto analizar los referentes teóricos esenciales, permiten diseñar la superación de los docentes, en aras de promover un salto cualitativo superior en sus modos de actuación. o por distintas razones entre las cuales se connota la vocación, la viabilidad en el ingreso y la promoción de la carrera 


\section{INTRODUCCIÓN}

$\mathrm{L}$

os centros educativos y la sociedad deben prepararse para afrontar los grandes retos científico- tecnológicos de la globalización, las comunicaciones y el manejo de la información. La sociedad actual denominada Sociedad del conocimiento, reconoce la importancia cada vez más creciente de la información como fuente para el desarrollo, constituyéndose así la sociedad de la información en una parte importante de ese proceso.

Muchas investigaciones en estas últimas décadas han ofrecido aportes científicos referidos al uso de las TIC,s y su impacto en la educación, así como su influencia en el aprendizaje de los estudiantes. Se destacan en el plano internacional: Chadwick (1987), Salomón( 1987), Vaquero( 1997) Pérez Marqués (2008- 2010- 2014) este último investigador europeo, destaca la necesidad de utilizar las TIC,s desde un enfoque didáctico y pedagógico, lo cual ha tenido un impacto extraordinario en la educación superior.

En la región, los investigadores Castellanos, D (2000), R L Martínez ( 2003), García, F. ( 2005), G Cervantes ( 2006), Zilverstein, S ( 2007) García, O ( 2010), Torres, A ( 2014), han ofrecido diferentes concepciones, modelos teórico -metodológicos del aprendizaje con el uso de la tecnología y se coincide en destacar la importancia de la eficiente dirección del proceso de enseñanza aprendizaje al emplearla como instrumento mediador y medio de enseñanza, así como la necesidad del diseño de diversos entornos virtuales, que se visualizan en los paradigmas de programación visual en la que confluyen la programación orientada a objetos y guidas por eventos en una interfaz gráfica para el usuario.

En Ecuador se connotan los estudios de Samaniego, F Giovanni ( 2009) y en la región de Cuenca Flanklin Miranda Realoe, ofreció en la universidad donde labora una maestría online acerca de cómo integrar didácticamente las NNTT en los entornos de cualquier carrera universitaria.

Los criterios abordados por dichos autores respecto al uso de las TIC,s evidencian la necesidad de profundizar en el enfoque de los recursos de aprendizaje en los entornos virtuales, en función de aprovechar de forma eficiente sus potencialidades en el desarrollo integral de la personalidad de los estudiantes, a través de la formación de una cultura tecnológica.

\section{Fundamentos epistemológicos.}

Por consiguiente la realidad educativa apunta hacia la necesidad de promover una correcta actualización de estos recursos por los docentes, desde lo teórico, pedagógico y didáctico, pues no todos dominan las TIC,s y logran interactuar con ella en el proceso de enseñanza aprendizaje. Para ello, se requiere de una superación permanente y continua que posibilite transformaciones en sus modos de actuación, con énfasis en la Educación superior, de forma que accedan a una contextualización en correspondencia con la carrera.

Los entornos virtuales se han convertido en una vía integradora y didáctica para ofrecer tratamiento al proceso de enseñanza aprendizaje en los centros educacionales, donde el estudiante requiere nuevas alternativas para su profundización en cada una de sus asignaturas.

En este sentido la carrera de Técnico Superior de enfermería que se estudia en el Instituto Superior Tecnológico Bolivariano de Tecnología de Guayaquil, requiere retomar las TIC,s como recurso indispensable para lograr una mejor formación profesional de sus egresados.

La formación es la categoría que promueve la relación entre los procesos instructivos y educativos en el sistema educacional, el cual se revela dentro de un sistema planificado, organizado y dirigido a lograr cambios en la personalidad del que estudia. Desde esta posición cobra particular importancia el proceso de formación profesional en cada una de las carreras de los Institutos Superiores Tecnológicos en el Ecuador.

En el ámbito de esta temática, muchos autores han investigado como: Álvarez de Zayas y Rita Marina (1995); Achiong, G (2005); Bautista, J (1997); Fuentes, H. (2000, 2002 y 2005); García, G; Paz, I (2005) Horruitiner, P (2008) entre otros, quienes destacan que esta categoría pedagógica posibilita el logro de una formación con calidad, como exige la sociedad a los centros de Educación Superior. Por tanto este término formación, incluye lo instructivo, educativo, metodológico y didáctico a través de este proceso.

Esa instrucción que requiere el estudiante se desarrolla a través de la categoría enseñanza, revelándose como un conjunto de procesos que promueven, desarrollan y fortalecen las facultades de los estudiantes y de los docentes, para aprender y enseñar. Para ello, se necesita de una comunicación mediática realizada (en un primer momento) por el docente facilitador de conocimientos y los estudiantes fuentes de conocimientos, de forma que se promuevan los procesos de comunicación y las facilidades o recursos para el aprendizaje. 
De modo que se asume que la formación "es la actividad organizada dirigida por el maestro para que los educandos se apropien de conocimientos, hábitos, habilidades y valores". Chávez, J (2006: 4), aspectos esenciales para una adecuada formación.

Por su parte el aprendizaje es considerado como un proceso dialéctico de apropiación de los contenidos y las formas de conocer, hacer, convivir y ser construidos en la experiencia socio histórico, lo cual posibilita en el estudiante la reflexión de sus ideas y su transformación en la actividad práctica.

Las categorías antes mencionadas no pueden analizarse al margen del desarrollo, que es el proceso de transformación de cualidades, actitudes, aptitudes, valores que contribuyen a perfeccionar a un individuo, desde su formación, ya sea individual o social.

Estas razones generales promueven el análisis de la necesidad que tiene el docente de la formación sistemática, que les posibilite un cambio de las potencialidades y facultades personales dentro de la organización institucional escolar. De esta manera, una forma de lograr el desarrollo humano es provocar un cambio que estimule, fomente y active el crecimiento del hombre, en lugar de mantenerlo pasivo, de modo que se convierta en un agente educativo consciente y dirigido.

Para ello, la teoría socio histórico cultural de Vigostky, destaca la importancia que cobran las categorías actividad - comunicación desde lo filosófico y psicológico en el proceso de apropiación de la cultura heredada, y que se transmite por el docente en el proceso de enseñanza aprendizaje. Solo así la formación de los sujetos sociales, en este caso los estudiantes, revela su carácter dialéctico y bilateral en plena relación maestro alumno, alumno, alumno en un proceso de construcción de saberes.

Estas consideraciones actuales acerca de promover la integración didáctica en el proceso de enseñanza aprendizaje, abre un amplio perfil en las universidades y tecnológicos de la Educación Superior, para la preparación teóricodidáctico- metodológica, del docente en aras de alcanzar su perfeccionamiento, donde cobra particular importancia la superación de los docentes, por ser ellos, los encargados de formar egresados de mayor calidad.

Desde esta óptica, la formación profesional incluye todos aquellos estudios y aprendizajes encaminados a la inserción, reinserción y actualización laboral, cuyo objetivo principal es aumentar y adecuar el conocimiento y habilidades de los actuales y futuros trabajadores a lo largo de la vida. Actualmente en la mayoría de países se le conoce como Educación y Formación Profesional, comprende las acciones formativas que capacitan para el desempeño cualificado de las diversas profesiones, favoreciendo el acceso al empleo, la participación activa en la vida social, cultural y económica, y la cohesión social.

En un sentido integrador, incluye las enseñanzas propias de la formación profesional del sistema educativo y de la formación profesional para el empleo, en ambos casos en la perspectiva de la adquisición y actualización permanente de las competencias profesionales. El autor de la investigación enfatiza que la formación del profesional garantiza:

- El aprendizaje, el dominio, las formas de proceder, de ser y de valorar a los estudiantes.

- El desarrollo de habilidades y capacidades profesionales
- La construcción de saberes y el desarrollo de significados y sentidos profesionales en formación
-

\section{La estimulación de modos de actuación profesional coherentes y relevantes desde lo personal y social.}

Es decir, si el docente demuestra modos de actuación profesional, es capaz de promover recursos de aprendizajes, los cuales estimulan el desarrollo de habilidades y dominios cognitivos e instrumentales en los estudiantes, de modo que adquiera significados y nuevos sentidos en sus modos de actuación.

En este sentido en estas últimas décadas ha cobrado significación la educación Técnica y profesional, no solo por las necesidades de mercado, si no como alternativa de palear una remuneración menos costosa y un trabajo mucho más rápido en cuanto a niveles de estudio, con énfasis en aquellos jóvenes con mayores necesidades económicas. Estas razones han abierto una expectativa desde lo macro social a las carreras tecnológicas en el país, considerándose desde la Ley orgánica establecida, la necesidad de formar a los jóvenes a partir de las necesidades sociales y lograr una formación de calidad y calidez. 
Es decir, si el docente demuestra modos de actuación profesional, es capaz de promover recursos de aprendizajes, los cuales estimulan el desarrollo de habilidades y dominios cognitivos e instrumentales en los estudiantes, de modo que adquiera significados y nuevos sentidos en sus modos de actuación.

En este sentido en estas últimas décadas ha cobrado significación la educación Técnica y profesional, no solo por las necesidades de mercado, si no como alternativa de palear una remuneración menos costosa y un trabajo mucho más rápido en cuanto a niveles de estudio, con énfasis en aquellos jóvenes con mayores necesidades económicas. Estas razones han abierto una expectativa desde lo macro social a las carreras tecnológicas en el país, considerándose desde la Ley orgánica establecida, la necesidad de formar a los jóvenes a partir de las necesidades sociales y lograr una formación de calidad y calidez.

Por ello, se exige a los docentes de estos centros universitarios, la calificación indispensable para cubrir los niveles de desempeño que se requieren alcanzar, donde se revela la necesidad de la contextualización de los contenidos y los enfoques didácticos que promuevan modos de actuación superior. Asimismo la necesidad de dominar el perfil de la carrera donde trabaja en aras de perfeccionar su labor a partir de estos objetivos.

El tecnólogo de salud requiere cumplir en su formación con los siguientes aspectos: el Técnico Superior de Enfermería podrá desempeñarse, bajo supervisión profesional de la licenciada en enfermería, tanto en el área pública como privada y en los tres niveles de atención, dentro del país y fuera de él, diferenciado en conducta, actitudes habilidades y aptitudes que requiere un técnico en enfermería.

El egresado debe ser un profesional preparado para la demanda competitiva de la actualidad con solidez científica, habilidades, destrezas, actitudes y valores que le permitan tomar decisiones en la solución de problemas de manera multi e interdisciplinaria; convirtiéndose así en un profesional confiable para el equipo de salud. Aplicar y generar el auto cuidado, nuevos conocimientos y el uso de tecnología que incidan en la salud-enfermedad del individuo, familia y comunidad en las diversas etapas de la vida.

Para lograr los objetivos de dicho proceso formativo asume gran importancia la preparación del docente, lo que exige el perfeccionamiento constante de su superación. La superación del docente debe centrarse en la adquisición de competencias que permitan una integración de los recursos TIC,s en función de las necesidades contextuales al combinar distintos enfoques: información, colaboración y aprendizaje.

Una de las formas de la superación del docente es la formación continua, la que constituye el conjunto de acciones formativas que se desarrollan para mejorar tanto las competencias y cualificaciones de los profesionales en formación, como la recalificación de los profesionales ocupados, que permitan compatibilizar la mayor competitividad de las empresas con la formación individual del profesional.

Desde esta perspectiva entonces la formación continua garantiza la actualización de los conocimientos de los profesionales y la permanente mejora de su calificación, así como incentivar en su trabajo diario su motivación profesional, lo que potencia la capacidad de los profesionales para su desempeño en relación con el beneficio individual, social y colectivo. Los distintos modelos teóricos y estándares presentados ofrecen un marco para el diseño, selección, análisis y evaluación de recursos TIC, s que debe formar parte de la capacitación permanente de los docentes y de la adquisición de competencias de aprendizaje autorregulado.

Desde el punto de vista psicológico la competencia es considerada "como una configuración psicológica que integra componentes cognitivos, metacognitivos, motivacionales y cualidades de personalidad, en estrecha unidad funcional, autorregulando el desempeño real y eficiente del individuo en una esfera específica de la actividad humana", (González, M 1999:28) en correspondencia con el modelo de desempeño deseable socialmente construido en cada contexto histórico concreto.

Pero en este sentido es justo reconocer, que no ha existido en lo metodológico dentro de los Institutos superiores Tecnológicos un trabajo sistemático de superación a los docentes de forma planificada y establecida, de modo que se ofrezcan referentes teórico- prácticos para su perfeccionamiento, lo que se convierte en una fisura de la investigación.

Por tales razones, resulta esencial considerar las competencias profesionales como "el conjunto de comportamientos socio-afectivos y habilidades cognoscitivas, psicológicas, sensoriales motoras que permiten llevar adecuadamente, un papel, una función, una actividad o una tarea". González Rey, (1999:45).

De modo que es una capacidad de acción eficaz frente a una gama de situaciones que se llega a dominar, porque se dispone tanto de los conocimientos necesarios como de la capacidad para movilizarlos eficazmente, en un tiempo oportuno para identificar y resolver problemas reales. 
Homero Fuentes en sus investigaciones enuncia que la competencia profesional, "constituye el saber, el hacer y el ser que se apropia un sujeto en aras de desempeñarse laboralmente, profesionalmente y socialmente. Es, en el lenguaje didáctico la integración de los núcleos o invariantes de conocimientos, de invariantes de habilidad y habilidades generalizadas y de valores profesionales y sociales." (Fuentes, H 1998:56)

Las competencias profesionales son la expresión de los contenidos teóricos e instrumentales prácticos, que permiten mostrar un adecuado desempeño profesional. Por consiguiente, si se asumen nuevos modos de desempeño, se necesita desarrollar una competencia profesional entendida como el " resultado de la integración, esencial y generalizada de un complejo conjunto de conocimientos, habilidades y valores profesionales, que se manifiesta a través de un desempeño profesional eficiente en la solución de los problemas de su profesión, pudiendo incluso resolver aquellos no predeterminados". (Forgas, J. 2003: 38)

La investigación al asumir estas aristas requeridas para el desarrollo de un docente competente, precisa que la superación profesional debe ser de forma sistemática, continua y que revele un alto nivel de actualización de los avances de la ciencia y especialmente de la tecnología, las cuales deben estar en función del perfeccionamiento del proceso de enseñanza aprendizaje en la formación del Técnico Superior de Enfermería.

En este sentido los referentes teóricos acerca de la filosofía y la psicología como sustentos teóricos para la superación de los docentes, valoran la teoría de la actividad como parte de la teoría del conocimiento, esencial en el proceso de enseñanza aprendizaje y en la formación continua del docente para su mejor desempeño profesional. Dentro de ella, se revela la actividad cognoscitiva y valorativa donde tanto docentes como estudiantes, interactúan en el proceso de enseñanza aprendizaje para transformar sus modos de actuación, a través de la comunicación eficaz, lo que corrobora su desempeño.

Por tanto la comunicación dentro de la actividad cobra particular significación en el contexto áulico en plena relación con los docentes y estudiantes y entre los propios discípulos. Desde la Psicología se parte de la teoría histórica cultural de Vygotsky acerca del desarrollo de los procesos psíquicos superiores, como resultado de la interacción social y su concreción en el condicionamiento social de la superación profesional en entornos virtuales, donde cobran particular interés, los procesos lógicos del pensamiento análisis y síntesis, inducción y deducción y el desarrollo de las habilidades superiores valorar, generalizar, argumentar entre otras, vitales todas para la apropiación de los conocimientos por los estudiantes.

Como referente pedagógico y didáctico se asume la teoría de educación de avanzada de Julia Añorga (Cuba) que concibe la misma como:

"esfuerzo sistemático planificado para el desarrollo de experiencias de aprendizaje que permita una actuación adecuada en una actividad o rango de actividades. Estas experiencias pueden tener carácter escolarizado o no, llevarse a cabo en instituciones especializadas o en la propia organización y actuar sobre el conocimiento, las habilidades, las actitudes o cualquier combinación de ellas". (Añorga, J. 1995: 89).

Esta autora destaca la integración de la formación continua y permanente del profesional para alcanzar la excelencia formativa. Se consideran también los fundamentos de la enseñanza aprendizaje desarrolladora de Doris Castellanos y los fundamentos pedagógicos acerca de la inserción de los entornos virtuales en la formación continua de los docentes (Ulises, Mestre: 1999:68).

Por consiguiente esta conceptualización del tecnólogo, en las actuales modalidades de instrucción y formación ofrecida por la Universidad vienen de la mano con el uso de las TIC,s en el contexto académico donde se tiene en cuenta el entramado de Universidad 2.0 el cual se está introduciendo en las aulas de educación superior

Muchos son los investigadores que expresan en sus aportes los referentes teóricos significativos para la incorporación de las TIC,s en el proceso de enseñanza aprendizaje. Pere Marqués, ( 2010), Toro, M: (2010), Pedraza, M ( 2014 en el plano europeo y en el plano regional Frómeta, E ( 2011), García, O (2012) entre otros.

Estas consideraciones estudiadas revelan la necesidad de potenciar nuevos modelos pedagógicos, entornos virtuales de colaboración con contenido digital accesible que se pueda compartir e intercambiar». El profesorado y el alumnado universitario no pueden verse como meros consumidores de información, sino también como participes de la misma y creadores de conocimiento. Castaño y Palazio (2007, p. 9).

Se evidencia, entonces la necesidad de abandonar las formas tradicionales de enseñanza en los centros universitarios y se concibe un nuevo concepto: la Universidad 2.0, en función del nivel de integración que ella realiza con las TIC,s donde el dominio de estas herramientas constituye el uso eficaz y se percibe como indispensables para toda la comunidad universitaria Pedreño, (2009) y Pere, Marqués, (2010). 
Estas razones permiten al alumno concentrarse en su aprendizaje y dotan al profesor de herramientas suficientes para la transmisión de conocimientos y el desarrollo de competencias y habilidades.

Se trata de integrar las TIC en el proceso metodológico y didáctico de la educación superior, convirtiéndose en herramientas fundamentales para apoyar la docencia en nuevos entornos formativos, para facilitar el aprendizaje y el logro de competencias del estudiante universitario, aunque para ello, sea necesario que el docente requiera una formación continua que le ofrezca la adquisición de competencias para su mejor nivel de desempeño profesional.

Este análisis posibilita arribar a consideraciones finales.

La utilización de las TIC,s en el proceso de enseñanza aprendizaje requiere de un superación continua de los docentes, de modo que se apropien de las herramientas teórico- didácticas y pedagógicas para aprovechar las potencialidades que posee la tecnología para el aprendizaje de los estudiantes en las carreras tecnológicas, con énfasis en el Técnico Superior de enfermería.

Esta contextualización por parte del docente resulta de vital importancia, pues no siempre está preparado para usar las TIC a partir de todas sus variantes: blog, aula virtual, página web, plataforma educativa, de modo que promueva el desempeño profesional y la calidad en la formación del estudiante durante el proceso de enseñanza aprendizaje, lo que se revertirá en sus modos de actuación y en la apropiación de sus conocimientos para la vida. 


\section{BIBLIOGRAFÍA}

ADDINE FERNÁNDEZ, FÁTIMA ET AL. (2005*). Didáctica: Teoría y Práctica. Editorial Pueblo y Educación. La Habana.

Principios para la Educación del proceso pedagógico. Editorial Pueblo y Educación. La Habana, 2003.

ÁLVAREZ DE ZAYAS, CARLOS. (1994). Didáctica. Editorial Pueblo y Educación. La Habana.

ALDAS MARIBEL ET AL. (2013). La educación a distancia y virtual en Ecuador: una realidad universitaria. Universidad Técnica particular de Loja. 1 edición. Junio.

AÑORGA, J. (1995). Teoría de los sistemas de superación. La Habana: Editorial pueblo y Educación. .

BRAVO RAMOS, JUAN LUIS. (2015). Los medios de enseñanza: Clasificación selección y aplicación. Consultado 20 de octubre 2015. en http\} www.sav.us.es, pixelbi tartículos 24. N24art 2009 htm

BRICEÑO MAGALLY. (2008). El profesor y la integración didáctica en la enseñanza universitaria. Magally Briceño y Migdy Chacón. En Revista Paradigma. Vol. 29. Venezuela.

DÍAZ ELIANA. (2008).Los juegos como recursos didácticos. Video. Consultado el 8 de septiembre 2014 blogspot. com

CHÁVEZ RODRÍGUEZ, JUSTO ET AL. (2003). Un acercamiento necesario a la Pedagogía General. ICCP. La Habana.

HORRUITINER SILVA, PEDRO. (2009). La universidad cubana: modelo de formación. Editorial Pueblo y Educación. La Habana.

FERNÁNDEZ DÍAZ, ELIA et al. (2012). La formación permanente del profesorado en el uso innovador de las TIC: una investigación en Infantil y primaria. En Revista de Currículo y formación del profesorado. Vol 16 \# 2 mayo agosto.

FUENTES GONZÁLEZ, HOMERO. Didáctica de la Educación Superior. Editorial Pueblo y Educación. La Habana, 2008.

FUENTES GONZÁLEZ, HOMERO. (2006). La epistemología de la investigación educativa. Colombia.

GONZALO LEÓN, SERRA et al. (2013). Evolución de los profesionales TIC en la sociedad del conocimiento. Consultado 2 de febrero 2013.En www.coit.es/pob/ficheros/pafet-2-0 e2b0b00.pdf.

GUANIPA PÉREZ. MARÍA. (2007). Formación permanente del docente en el marco de las tecnologías y la comunicación. María Guanipa y Janno M Pérez. En Revista Ciencias de la Educación. Vol 1 \# 30 . Valencia. España.

MARQUÉS, GRAELLS, P. Modelos didácticos de aprendizaje en grupo y aprendizaje colaborativo con TIC. Consultado 2 de agosto de 2014. En www. Monografía. com.

MELLADO DURÁN, ENCARNACIÓN et al. (2011). Las TIC como herramienta fundamental de la formación permanente en la Universidad de Sevilla. En Revista de Medios y Educación \# 39 julio. .

MILIÁN VÁZQUEZ, PEDRO MIGUEL. (2011). Tesis doctoral en opción al Título académico de Doctor en Ciencias Pedagógicas. Farmacología y Didáctica para la Salud. Cienfuegos. Cuba. .

MORÉN OLIVOS, TIBURCIO. Didáctica de la Educación Superior: nuevos desafíos en el siglo XXI. Consultado 21 de agosto 2014. Sitio www//monografía/ Universidad metropolitana/ México.

PARRA VIGO, ISEL. (2003). Modelo didáctico para contribuir a la dirección del desarrollo de la competencia didáctica del profesional de la educación en formación inicial. Tesis en opción el Título académico de Doctor en Ciencias de la Educación. La Habana, Cuba.

PEDROZA MARCOS, ANTONIO. (2014). Los entornos virtuales de enseñanza aprendizaje. Extraído 21 de agosto 2014. Http://www. Centros/revistas/index.html. 
PEDRAZA GONZALEZ, XENIA. (2013). La superación profesional: una forma de superación permanente para los docentes. 